\title{
Dexamethasone but not the equivalent doses of hydrocortisone induces neurotoxicity in neonatal rat brain
}

\author{
Yangzheng Feng', Praveen Kumar', Junming Wang ${ }^{2}$ and Abhay J. Bhatt'
}

BACKGROUND: The use of dexamethasone (Dex) in premature infants to treat or prevent chronic lung disease adversely affects neurodevelopment. Recent clinical studies suggest that hydrocortisone $(\mathrm{HC})$ is a safer alternative to Dex. We compared the effects of Dex and HC on neurotoxicity in newborn rats.

METHODS: Rat pups of a neurodevelopmental stage equivalent to premature human neonates were administered Dex or $\mathrm{HC}$ either as a single dose on postnatal day (PD) 6, repeated doses on PD 4 to 6 or tapering doses at PD 3 to 6 by i.p. injection. Brain weight, caspase-3 activity, and apoptotic cells were measured at PD 7; learning capability, memory, and motor function were measured at juvenile age.

RESULTS: Dex decreased both body and brain weight gain, while HC did not. Tapering and repeated doses of Dex increased caspase-3 activity, cleaved caspase-3 and terminal deoxynucleotidyl transferase dUTP nick end labeling (TUNEL)-positive cells but $\mathrm{HC}$, except at high doses, did not. Dex impaired learning and memory capability at juvenile age, while the rats exposed to HC showed normal cognitive behavior.

CONCLUSION: $\mathrm{HC}$ is probably safer to use than Dex in the immediate postnatal period in neonatal rats. Cautious extrapolation of these findings to human premature infants is required.

D examethasone (Dex) has been shown to reduce the incidence and severity of chronic lung disease (CLD) in the premature infants; however, its use has been reported to adversely affect neurodevelopment, neurocognitive, and behavioral development and associated with higher rates of cerebral palsy $(1,2)$. Animal studies have also demonstrated that Dex impairs brain function, fetal growth, and neurodevelopment $(3,4)$. These concerns have highlighted the need to find a safer alternative to Dex for the treatment of CLD. Recently, clinical studies reported that postnatal hydrocortisone (HC) treatment for CLD may have fewer or no adverse effects on neurodevelopment and has been suggested to be a safer alternative to Dex (5-7) although these studies did not show any benefits of HC against CLD (8). Huang et al. (9) showed that neonatal Dex treatment, but not $\mathrm{HC}$, altered hippocampal synaptic plasticity and associative memory formation in later life in rats. In contrast, Dex and HC increased cell death and induced caspase-3 activation in a similar dose-dependent manner in cultures of embryonic chicken cerebellar granule cells (10). To explore this important issue further, we performed the present study to evaluate whether different regimens of $\mathrm{HC}$ cause neurodegeneration in newborn rats and compared the results to neurotoxic effects of Dex administration. We also tested learning and memory capability and motor function at juvenile ages.

\section{RESULTS}

\section{Dex, But Not HC, Decreased Body Weight Gain}

Neonatal Dex tapering doses (DexTD) exposure significantly decreased body weight gain compared with the HC tapering doses (HCTD) and vehicle groups $(P<0.01$, Figure 1a). Treatment with Dex repeated doses (DexRD) and Dex single dose (DexSD) also significantly decreased body weight gain compared with the corresponding $\mathrm{HC}$ and vehicle groups $(P<0.01$, data not shown). Conversely, neonatal HCTD exposure, in all three dose (lower, equivalent to Dex, and higher dose) groups, did not affect body weight gain compared with the corresponding vehicle groups (Figure 1a). Similarly, neonatal HC repeated doses (HCRD) and HC single dose (HCSD) exposure also did not affect body weight gain (data not shown).

\section{Dex, But Not HC, Decreased Brain Weight}

Compared with pups in HCTD and vehicle groups, brain weights in DexTD-treated pups were significantly decreased at PD7, $1 \mathrm{~d}$ after treatment $(P<0.01$, Figure $1 \mathrm{~b})$. However, treatment with HCTD, across all three dose groups did not affect brain weight gain compared with vehicle groups (Figure 1b). Treatment with DexRD also significantly decreased brain weight compared with the corresponding vehicle groups but HCRD did not (data not shown).

\section{Dex, But Not Equivalent Doses of HC Increased Caspase-3 Activity}

There was a significant increase in caspase- 3 activity at PD7, $1 \mathrm{~d}$ after treatment with DexTD $(P<0.01$, Figure 2a), DexRD and a high dose $(30 \mathrm{mg} / \mathrm{kg})$ of $\operatorname{HCRD}(P<0.01$, Figure $2 \mathrm{~b})$ compared with vehicle groups. No change was noted in caspase-3 activity at any dose of HCTD (Figure 2a). Single doses of Dex or HC did not change caspase-3 activity (Figure 2c). 


\section{Dex But Not Equivalent Dose of HC Increased TUNEL-Positive Cells}

There was a significant increase in TUNEL-positive cells in the cortex and hippocampus after DexTD as well as in cortex following higher doses $\mathrm{HCTD}\left(\mathrm{HCTD}_{\mathrm{HD}}\right)$ compared with the corresponding vehicle groups $(P<0.01$, Figure 3$)$. On the contrary, HCTD in lower doses $\left(\mathrm{HCTD}_{\mathrm{LD}}\right)$ or middle (equivalent
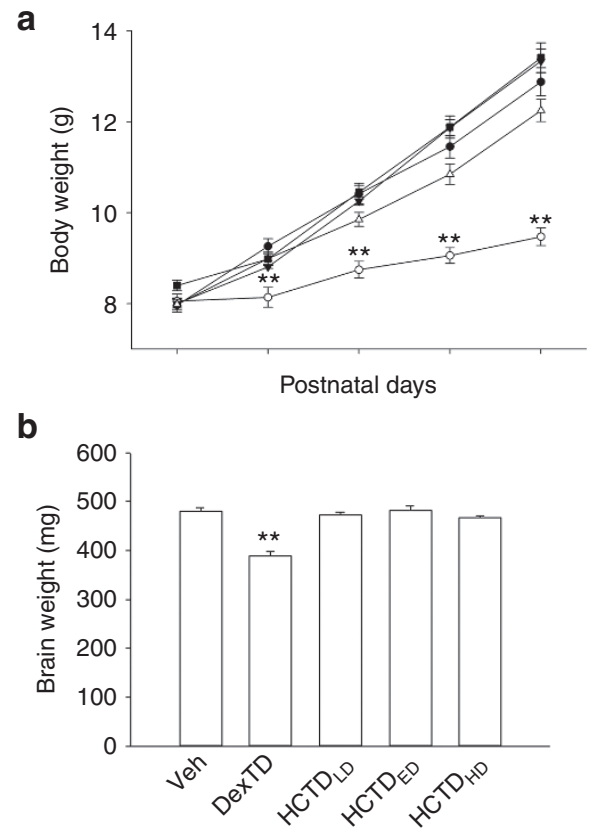

Figure 1. DexTD, but not hydrocortisone tapering doses (HCTD) decreased body and brain weight in rat pups. (a) The effect of DexTD (open circles) and HCTD (triangles and squares) on body weight gain in rat pups. HCTD, at any dose had no effect on body weight gain compared with the vehicle (closed circles). DexTD-treated rats had lower body weight gain than the vehicle and HCTD. Data are presented as mean \pm SEM. $n=6 /$ group, ${ }^{* *} P<0.01$ vs. vehicle and HCTD. (b) The effect of DexTD and HCTD on brain weight in rat pups. Brain weight was measured at PD7. HCTD at any dose had no effect on brain weight compared with vehicle. Treatment with DexTD significantly decreased brain weight compared with HCTD and vehicle. Data are presented as mean \pm SEM. $n=8$ / group, ${ }^{* *} P<0.01$ vs. HCTD and vehicle. to DexTD) doses $\left(\mathrm{HCTD}_{\mathrm{ED}}\right)$ did not increase TUNEL-positive cells (Figure 3 ).

\section{Dex Increased Cleaved Caspase-3-Positive Cells But Equivalent Doses of HC Did Not}

There was a significant increase in cleaved caspase-3-positive cells in the cortex and hippocampus after DexTD as well as in the cortex following $\mathrm{HCTD}_{\mathrm{HD}}$ compared with the corresponding vehicle groups $(P<0.01$, Figure 4$)$. Conversely, $\mathrm{HCTD}_{\mathrm{LD}}$ or $\mathrm{HCTD}_{\mathrm{ED}}$ did not increase cleaved caspase-3-positive cells (Figure 4).

\section{Dex, But Not Equivalent Doses of HC Induced Learning and Memory Deficits}

Passive avoidance test results are shown in Figure 5. The number of electric foot shocks before rats found the safe board was significantly increased in the DexTD group (7.5 (5.5, 10), $P<0.01)$ but this increase was not seen in the $\mathrm{HCTD}_{\mathrm{ED}}$ group $(4(3,7.3))$ when compared with the vehicle group $(4(3$, 5 ), Figure 5a). DexTD reduced the retention latency to step down from the safe board $(48.5(33,112), P<0.05)$ as compared to the control $(120(110,120))$ but HCTD $_{\mathrm{ED}}$ did not $(120$ $(61,120)$, Figure 5b). Neither DexTD nor HCTD affected the motor coordination and balance of rats as determined by rotarod at PD27 and PD28 (Figure 5c,d).

\section{DISCUSSION}

In this study, we compared the effects different regimens of Dex and HC on the neonatal rat brain. We confirmed that Dex, at all doses, significantly decreased the gain in body and brain weight, while HC did not. Both tapering and repeated doses of Dex significantly increased caspase- 3 activity, cleaved caspase-3- and TUNEL-positive cells in the developing brain. Although, similar to DexTD, HCTD at high dose did cause increased TUNEL- and caspase-3-positive cells; HC, in doses equivalent to or lower than Dex, did not induce significant apoptotic changes. Our study further elucidates that Dex impaired learning and memory capability in later life, while the rats exposed to an equivalent dose of $\mathrm{HC}$ showed normal cognitive behavior. a

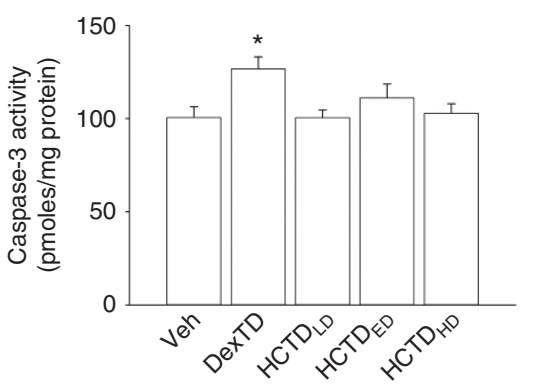

b

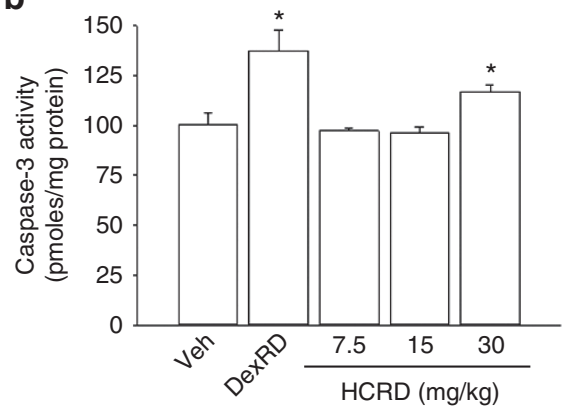

c

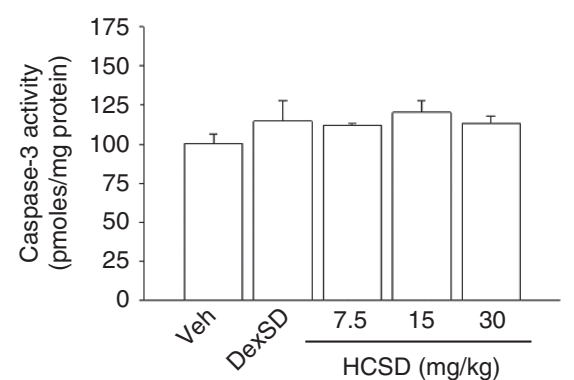

Figure 2. Effects of Dex and hydrocortisone $(\mathrm{HC})$ on caspase-3 activity in the pup brain cortex. Analyses were performed $24 \mathrm{~h}$ after treatment at PD7. Response to DexTD and HCTD (a), DexRD and HCRD (b), and the dose-response curve after different single doses of HC (c) are shown. DexTD and DexRD significantly increased caspase-3 activity compared with vehicle. HCRD at high dose $(30 \mathrm{mg} / \mathrm{kg})$ significantly increased caspase-3 activity compared with vehicle but middle and lower doses did not. HCTD and HCSD treatment at any dose or DexSD did not change caspase-3 activity compared with vehicle. Data are presented as mean \pm SEM. $n=5-13 /$ group, ${ }^{*} P<0.05$ vs. vehicle. HCTD, hydrocortisone tapering doses. 


\section{Articles | Fenget al.}
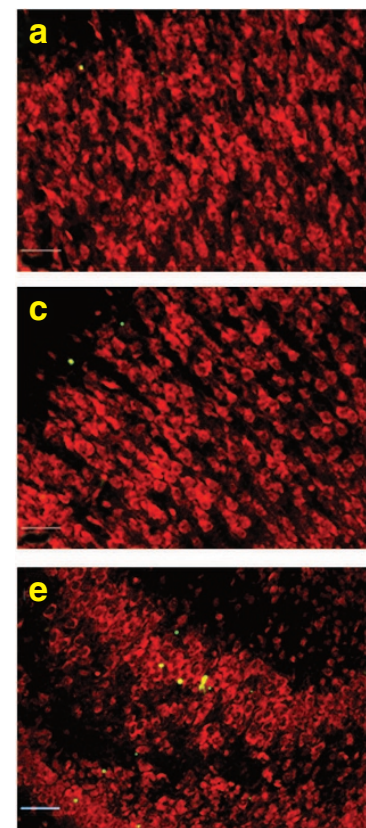
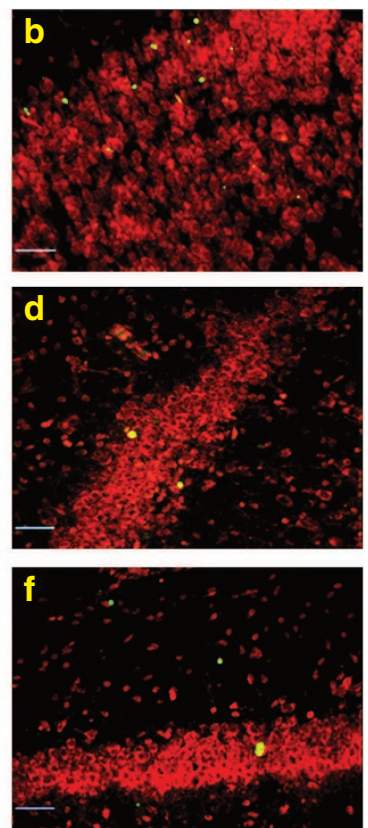

g

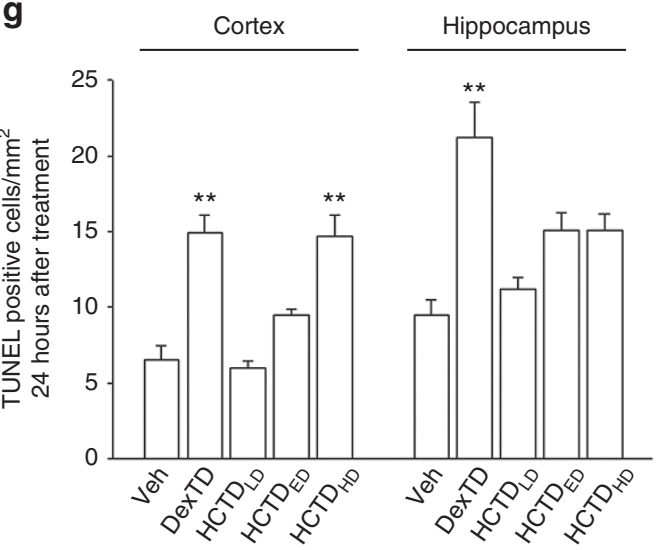

Figure 3. Immunofluorescence analysis of TUNEL-positive cells in cerebral cortex and hippocampus in the pup brain. Representative images of cortex and hippocampus in pup treated with vehicle $(\mathbf{a}, \mathbf{d}), \operatorname{DexTD}(\mathbf{b}, \mathbf{e})$, and $\operatorname{HCTD}_{\mathrm{ED}}(\mathbf{c}, \mathbf{f})$ are shown. TUNEL-positive nuclei are green and cells counterstained with propidium iodide (PI) are red. Vehicle- and HCTD-treated brain showed few TUNEL-positive cells, while treatment with DexTD increased TUNEL-positive cells. Quantification of the number of TUNEL-positive cells in the brain cortex and hippocampus are shown in (g). There was a significant

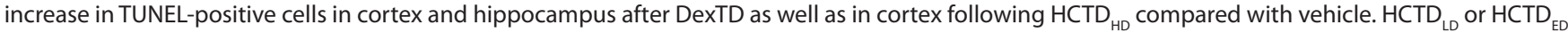
did not increase TUNEL-positive cells. Data are presented as mean \pm SEM. $n=8 /$ group, ${ }^{* *} P<0.01$ vs. vehicle. Images were captured using a $20 \times$ objective, scale bar $=50 \mu \mathrm{m}$. HCTD, hydrocortisone tapering doses.
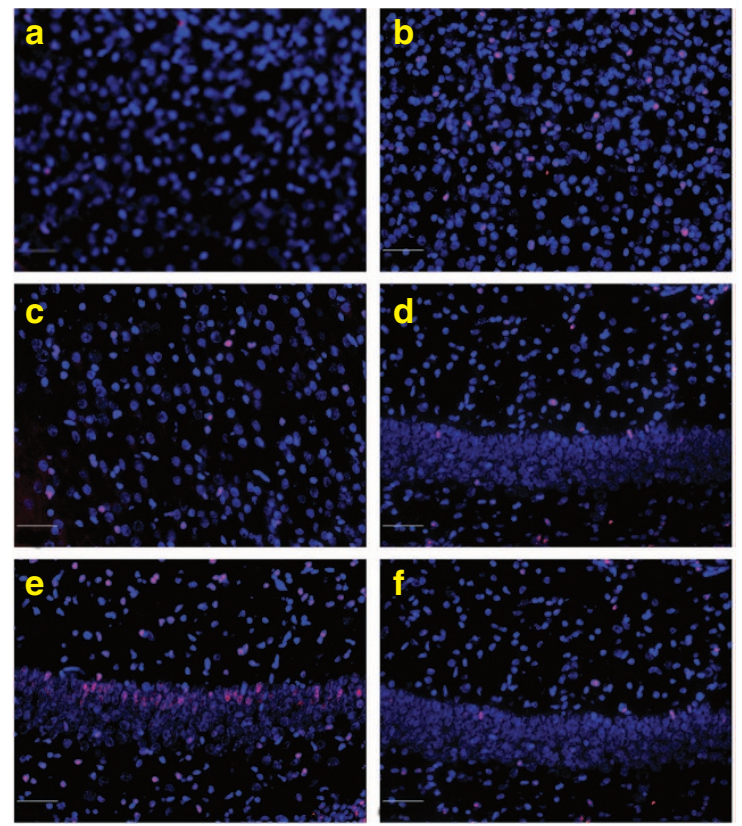

g

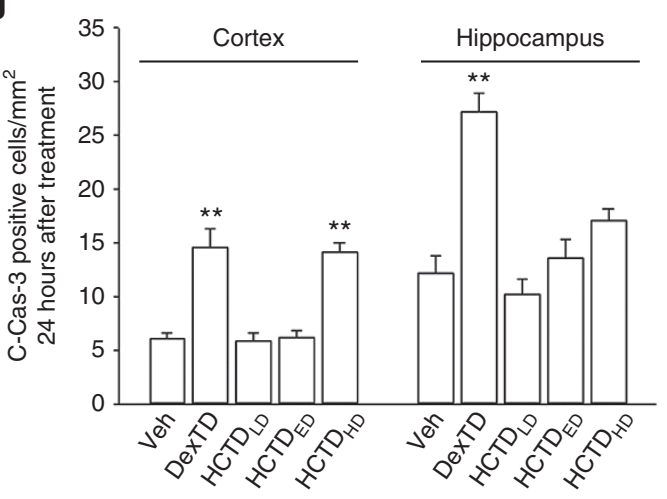

Figure 4. Immunofluorescence analysis of cleaved caspase-3 in cerebral cortex and hippocampus in the pup brain. Representative images of cortex $(\mathbf{a}-\mathbf{c})$ and hippocampus $(\mathbf{d}-\mathbf{f})$ in pup treated with vehicle $(\mathbf{a}, \mathbf{d})$, DexTD $(\mathbf{b}, \mathbf{e})$, and $\mathrm{HCTD}_{\mathrm{ED}}(\mathbf{c}, \mathbf{f})$ are shown. Cleaved caspase-3-positive cells are red and nuclei counterstained with DAPI are blue. Vehicle- and HCTD-treated brain showed very low levels of staining for cleaved caspase-3, while treatment with DexTD produced robust cleaved caspase-3 staining. Quantification of the number of cleaved caspase-3-positive cells in brain cortex and hippocampus are shown in (g). There was a significant increase in cleaved caspase-3-positive cells in cortex and hippocampus after DexTD as well as in cortex following $\mathrm{HCTD}_{\mathrm{HD}}$ compared with vehicle. $\mathrm{HCTD}_{\mathrm{LD}}$ or HCTD ED did not increase cleaved caspase-3-positive cells $(P>0.05)$. Data are presented as mean $\pm \mathrm{SEM} . n=8 /$ group, ${ }^{* *} P<0.01$ vs. vehicle. Images were captured using a $20 \times$ objective, scale bar $=50 \mu \mathrm{m}$. HCTD, hydrocortisone tapering doses. 
a

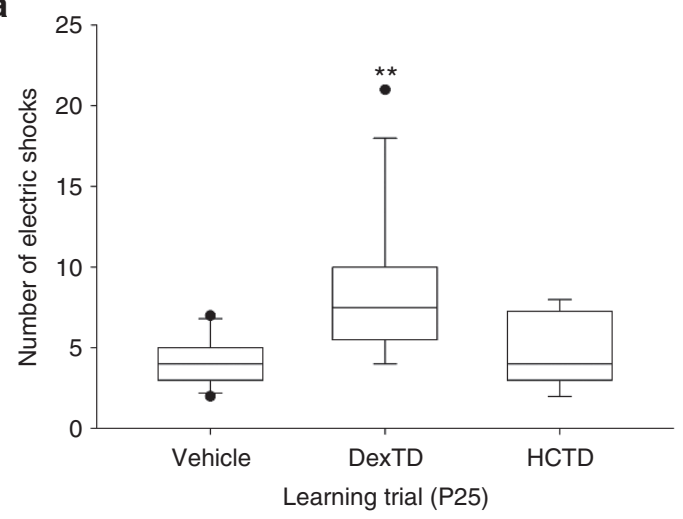

C

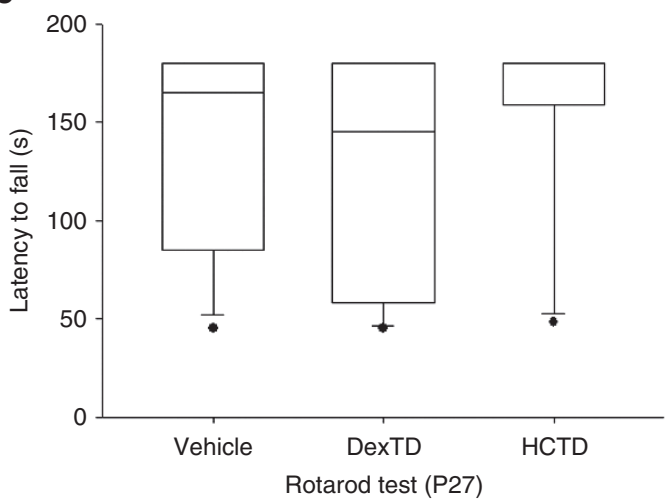

b

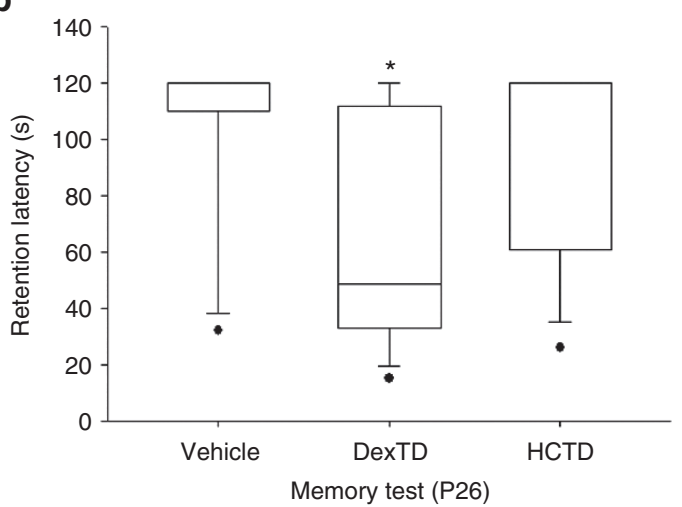

d

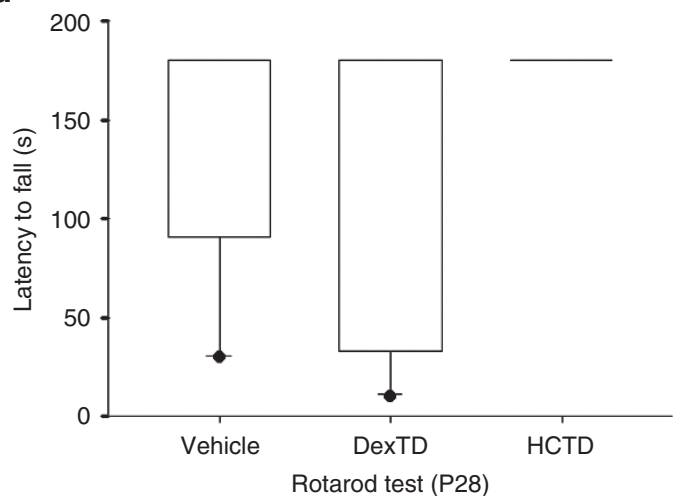

Figure 5. Neonatal DexTD, but not $\mathrm{HCTD}_{\mathrm{ED}}$, induced learning and memory deficits as determined by passive avoidance at PD25 and PD26 (a,b). Neonatal DexTD and HCTD treatment, did not affect coordinated motor ability as determined by rotarod at PD27-PD28 (c,d). Data are presented as median (25th percentile, 75 th percentile). $n=11-14$ rat pups for each group, ${ }^{*} P<0.05,{ }^{* *} P<0.01$ vs. vehicle. HCTD, hydrocortisone tapering doses.

In our previous studies, we have investigated and discussed the adverse effects of Dex on body weight and brain weight, caspase-3 activity, and apoptotic changes (11). The current report explores the differences between Dex and HC. We observed that HC did not reduce body weight or brain weight. These results are consistent with observations reported in human infants. In a retrospective study, a tapering course of Dex for 21 $\mathrm{d}$ was associated with reduced weight gain, but the weight gain of infants receiving HC therapy was comparable to controls, however head circumference in both treatment groups was lower after therapy (5). On the contrary, in a prospective study of preterm infants, neonatal $\mathrm{HC}$ did not reduce cerebral tissue volumes at term equivalent age as measured by a 3D-MRI (6). Furthermore, we showed that all three regimens of HCTD did not increase caspase-3 activity. However, repeated high doses of $\mathrm{HC}(30 \mathrm{mg} / \mathrm{kg})$ increased caspase- 3 activity. Additionally, similar to DexTD, HCTD at high dose did cause apoptotic changes in the form of increased TUNEL- and caspase-3positive cells. Tapering doses of HC in the middle or lower range did not induce apoptosis. In an in vitro study, Aden et al. (10) reported that HC has similar neurotoxic effects as Dex on neurons in the immature chicken cerebellum, but similar to another in vivo study (9) our results suggest that $\mathrm{HC}$ is safer than Dex. Our findings are also consistent with clinical studies. HC had no effect on brain growth, measured at term equivalent age after treatment of CLD (6). In another study involving
60 premature infants followed up at $8 \mathrm{y}$ of age, perinatal HC had no long-term effects on either neurostructural brain development or neurocognitive outcome (7). Our study showed that Dex exposure during the neonatal period impaired learning and memory capability at the juvenile age. These findings are in accordance with other studies. For example, tapering treatment with Dex in neonatal rats showed impaired performance in both the multiple and single trial hidden platform versions of the Morris maze that address both reference and working memory at adulthood, respectively (12). Animal performance in the visible platform version was normal which indicated that sensorimotor and motivational functions were not altered. Therefore, the authors concluded that impaired performance in hidden platform versions of the Morris maze clearly pointed to a cognitive deficit in Dex-treated rats (12). Furthermore, DeKosky et al. (13) examined behavior at PD60 after neonatal treatment, Dex-treated animals showed impaired acquisition and reversal of a spatial learning task in an open field water maze. These deficits are characteristically seen in animals with hippocampal damage. Many clinical studies have shown the adverse effects of Dex at school age $(1,2)$. Limited information is available about the neurodevelopmental outcome at adolescent age. Jones et al. (14) evaluated neurological, psychological, and educational outcomes at 13-17 y in subjects enrolled in a randomized, controlled trial of Dex in neonatal CLD. There was no clear difference between the Dex and placebo 


\section{Articles | Fenget al.}

group. However, the original study was not designed with enough power to evaluate the neurodevelopmental outcome at a later age, and there was a significant overlap of open-label use of Dex between the two groups. In summary, animal studies evaluating behavioural changes following glucocorticoids (GCs) exposure are done in older animals. But, clinical studies lack evidence of delayed effects at the later age. Consequently, we decided to use juvenile rats to evaluate behavior instead of older animals used by other researchers to find a bigger difference between Dex and HC if present. The present study showed that Dex did not affect motor function by rotarod tests. There were no adverse effects on coordination, balance, learning, and memory following $\mathrm{HC}$ treatment. These results are in agreement with the study of Huang et al. (9), which reported that Dex treatment alters the hippocampal synaptic plasticity and associative memory formation in later life but HC did not. Similarly, clinical reports show that HC-treated infants have significantly better school performance in comparison with their Dex-treated counterparts at 5-7 y of age (5).

Use of GCs in premature infants in clinical settings is controversial, beyond the scope of this article and discussed in detail elsewhere $(15,16)$. Briefly, HC in low doses is used for hypotension in extreme premature infants, while Dex and HC in different doses as well as for different durations are used for the prevention and treatment of CLD. Tapering doses of Dex used in the present study closely mimic a clinically relevant prolonged 42-d treatment regimen (3). The starting dose of $15 \mathrm{mg} / \mathrm{kg} \mathrm{HC}$ is almost equivalent to the glucocorticoid activity of $0.5 \mathrm{mg} / \mathrm{kg}$ Dex (17). In clinical settings, HC regimens include $5 \mathrm{mg} / \mathrm{kg} / \mathrm{d}$ for $1 \mathrm{wk}$, then tapering course over $3 \mathrm{wk}$ (6), or $2 \mathrm{mg} / \mathrm{kg} / \mathrm{d}$ for $2 \mathrm{~d}, 1.5 \mathrm{mg} / \mathrm{kg}$ for $2 \mathrm{~d}$ and $0.75 \mathrm{mg} / \mathrm{kg} / \mathrm{d}$ for 6 $\mathrm{d}$ (18) or $1 \mathrm{mg} / \mathrm{kg} /$ for $9 \mathrm{~d}$, then $0.5 \mathrm{mg} / \mathrm{kg} / \mathrm{d}$ for $3 \mathrm{~d}$ (16). The tapering course of $\mathrm{HC}$ in animal experiments was $5 \mathrm{mg} / \mathrm{kg}$ on $\mathrm{PD} 1,3 \mathrm{mg} / \mathrm{kg}$ on PD2, and $1 \mathrm{mg} / \mathrm{kg}$ on PD3 (9). We studied three tapering doses including higher, middle (equivalent to Dex), and lower doses as well as single and repeated doses of HC. The higher dose is a toxic dose which is over sixfold higher than the usual clinical doses. The middle dose (equivalent to Dex) is still higher than the usual doses and the lower dose are the closest to the currently used HC doses in infants. In the present data, the apoptotic neurodegeneration studies do not show any adverse effects after neonatal HC exposure even at doses higher than the usual doses used clinically.

Why is neonatal treatment with HC less neurotoxic than Dex? Potential reasons include their different GC activity, half-life, and receptor affinities. Dex has a higher glucocorticoid-mediated than mineralocorticoid-mediated activity and longer biological half-life compared with HC. HC, which is identical to native cortisol, can bind to both mineralocorticoid and glucocorticoid receptors or binds preferentially to the mineralocorticoid in the brain, whereas Dex binds only to glucocorticoid receptors or preferentially to glucocorticoid receptors (13). A report by Heine and Rowitch (19) on the complex interplay between GCs and the sonic hedgehog (Shh) signaling pathway in proliferating cerebellar granule neuron precursors in postnatal mice sheds light on the relative safety of $\mathrm{HC}$ in the developing brain. Shh signaling induced $11 \beta$-hydroxysteroid dehydrogenase type 2 (11ßHSD2), a NAD-dependent highaffinity enzyme. $11 \beta \mathrm{HSD} 2$ is involved in the local metabolic inactivation of endogenous GCs like HC into inert 11-keto derivatives but has no effects on synthetic GCs like Dex. Thus, $11 \beta \mathrm{HSD} 2$ antagonized the antiproliferative and apoptotic effects of 11ßHSD2-sensitive HC but not Dex on proliferating mouse cerebellar granule neuron precursors (19).

A cautious approach is needed before directly extrapolating our findings in newborn rats to human premature infants. Additionally, further animal studies evaluating the effect of HC on neurogenesis, myelination, and synapses would be helpful. While, the therapeutic efficacy of HC to treat or prevent CLD is debatable, the present studies and previous clinical reports serve to indicate that HC treatment is probably safer than Dex in the neonatal brain. Due to the concern of adverse effect on neurodevelopment, the use of Dex has dropped dramatically to $\sim 10 \%$ of the preterm infants at risk for CLD (20), which is associated with increased rate of severe CLD (21). Current evidence strongly suggests that the benefits of early or late postnatal corticosteroid treatment, particularly Dex, may not outweigh the known or potential adverse effects of the treatment $(8,22)$. Some clinicians have started to use HC, as an alternative for Dex, but there is a lack of literature supporting this practice; there are no placebo-controlled randomized trials that investigated the use of HC. A large multicenter trial investigating early prophylactic HC treatment in premature infants was stopped prematurely for concern for excess gastrointestinal perforation showed benefit of HC in a subgroup of infants born after chorioamnionitis (23), the decision debated by the experts $(24,25)$.

In conclusion, Dex but not the equivalent dose of $\mathrm{HC}$, significantly decreased the weight gain of body and brain, induced apoptosis in neonatal rats, and impaired learning and memory capability at juvenile ages. We recommend further animal experiments and also support a large, multicenter, randomized, placebo-controlled, prospective trial to study the efficacy as well as short and long-term safety of $\mathrm{HC}$ as a rescue therapy of CLD in human premature infants.

\section{METHODS}

\section{Animals}

All experiments were approved by the University of Mississippi institutional committee on animal use. Gestationally timed pregnant SpragueDawley rats ( $n=27$, Charles Rivers, Wilmington, MA) were housed in our animal unit and maintained in accordance with the US National Institutes of Health (Bethesda, MD) guidelines. According to different experiments, majority of pups were killed by cervical dislocation; brains were dissected, weighed, and stored at $-80{ }^{\circ} \mathrm{C}$ for caspase- 3 activity measurements; the rest of pups were anaesthetized and received transcardiac perfusion for TUNEL staining and immunohistochemistry analysis. In another experiment, pups were allowed to grow for 28 $\mathrm{d}$ for behavioral tests. Although the evidence suggests that sex is not a factor affecting brain weight and neurological outcome following neonatal Dex exposure at PD7 $(4,11)$, we divided male and female pups in similar numbers in DexTD, HCTD, and vehicle groups. We pooled data because the findings in males and female rats were similar.

\section{Drug Treatment}

The day of birth was designated as PD1. On PD2, each litter was culled to 10 or 12 pups per litter, sexed, and randomly divided to 
three groups: vehicle $(n=82), \mathrm{HC}(n=143)$, and Dex $(n=57)$ treated groups. Animals received one of the three different regimens of Dex phosphate (American Regent, Shirley, NY) or HC (Pharmacia \& Upjohn, NY). Rat pups in the DexTD group received Dex on PD3 to PD6 $(0.5,0.25,0.125$, and $0.06 \mathrm{mg} / \mathrm{kg} / \mathrm{d}$, respectively). Rat pups received HCTD in the three different ranges on PD3 through PD6: $\mathrm{HCTD}_{\mathrm{TD}}: 7.5,3.8,1.9$, and $0.9 \mathrm{mg} / \mathrm{kg} / \mathrm{d}$; HCTD $\mathrm{HD}_{1}: 15,7.5,3.8$, and $1.9 \mathrm{mg} / \mathrm{kg} / \mathrm{d}$ and $\mathrm{HCTD}_{\mathrm{HD}}: 30,15,7.5$ and $3.8 \mathrm{mg} / \mathrm{kg} / \mathrm{d}$, respectively. For repeat doses: animals received $0.5 \mathrm{mg} / \mathrm{kg} / \mathrm{d}$ of Dex (DexRD) or 5 , 15 and $30 \mathrm{mg} / \mathrm{kg} / \mathrm{d}$ of $\mathrm{HC}$ (HCRD) on PD4 to PD6, respectively. For single-dose groups, animals received $0.5 \mathrm{mg} / \mathrm{kg}$ of Dex (DexSD) or 15,30 , and $60 \mathrm{mg} / \mathrm{kg}$ of HC (HCSD) on PD6. Animals in the corresponding vehicle groups received equivalent volumes of saline. These doses and modes of administration were chosen from previous studies $(3,9,26)$. The typical total dose of $\mathrm{HC}$ of $5-20 \mathrm{mg} / \mathrm{kg}$ equates to $\sim 0.2-0.6 \mathrm{mg} / \mathrm{kg}$ of Dex (17). The anti-inflammatory effect of $20 \mathrm{mg}$ of $\mathrm{HC}$ is equivalent to $0.6 \mathrm{mg}$ of Dex (17).

\section{Measurement of Weights of Body and Brain}

Pups were weighed before, during and $1 \mathrm{~d}$ after treatment (PD7). Pup brains were weighed at $1 \mathrm{~d}$ after treatment (PD7).

\section{Measurement of Caspase-3 Activity}

Caspase-3 activity was measured as described previously (11) at $1 \mathrm{~d}$ after treatment at PD7 in all groups. The brain cortex was homogenized and caspase- 3 activity was measured using commercially available assay kits (Calbiochem, San Diego, CA) following the manufacturer's instructions. Caspase-3 activity was determined colorimetrically with a microplate reader at $405 \mathrm{~nm}$. A p-nitroanilide (pNA) calibration curve was established to quantify units of caspase- 3 activity. The protein concentration was determined by the method of Bradford (27).

\section{Preparation of Brain Specimens}

Brain specimens from DexTD, HCTD, and vehicle groups were prepared as described previously (11). At PD7, pups were anesthetized with pentobarbital and received transcardiac perfusion with icecold saline and then with $4 \%$ paraformaldehyde in PBS. Brains were removed and immersed in 10\% formalin and then cryoprotected and rapidly frozen by 2 -methylbutane chilled in liquid nitrogen. Coronal brain was cut into $10-\mu \mathrm{m}$-thick sections and stored at $-80{ }^{\circ} \mathrm{C}$ for future analysis.

\section{Immunofluorescence Analysis}

Apoptotic cell death was visualized using TUNEL staining and performed using ApopAlert DNA Fragmentation assay kit (Clontech, Mountain View, CA) for immunofluorescence according to manufacturer's instructions. Immunofluorescence analysis for cleaved caspase- 3 was performed under standard protocols as described by manufacturer's instructions. Antibodies included rabbit anti-cleaved caspase-3 (1:200; Cell Signaling Technology, Danvers, MA) and antirabbit IgG conjugated with Cy3 (1:200; Jackson immnoReseach, West Grove, PA).

\section{Cell Counting}

The numbers of TUNEL and caspase-3-positive cells were counted under fluorescent microscopy at a 200x magnification in the brain sections at the bregma level and the middle dorsal hippocampus level. For all brain sections, anatomical landmarks were identified according to the atlas of Paxinos and Watson (28). The number of positively stained cells from 10 different fields in frontal cortex sections was averaged for each brain. For convenience of comparison, the results were converted to cells per $\mathrm{mm}^{2}$.

\section{Learning and Memory Testing}

To test learning and memory capabilities in DexTD, HCTD ${ }_{\mathrm{ED}}$, and vehicle-treated rat pups, the method of passive avoidance was used as described by Fan et al. (29) at PD 25-26. The passive avoidance procedure consists of two sessions. In the first session (PD25), rats were trained in a step-down type of passive avoidance apparatus. The experimental chamber $\left(30 \times 30 \times 40 \mathrm{~cm}^{3}\right)$ was made of plexiglass. The floor of the chamber was made of parallel $2-\mathrm{mm}$-caliber stainless steel rods spaced $1 \mathrm{~cm}$ apart and connected with an electric shock generator. The safe part was a piece of wood board $\left(7.5 \times 16 \times 2 \mathrm{~cm}^{3}\right)$ placed at a corner of the chamber above the metal rods. Each animal was placed initially on the safe platform. When the rat stepped down onto the floor, it received a foot shock $(1 \mathrm{~s}, 0.5 \mathrm{~mA})$. Rats repeatedly stepped up and down and eventually remained on the board. The number of shocks required to retain an individual animal on the board for $2 \mathrm{~min}$ was recorded as a measure of acquisition of passive avoidance. The second session was carried out $24 \mathrm{~h}$ after the first session (PD26). The rat was placed on the safe board and steel rods were not connected with the electric shock generator. The retention latency, i.e., the time elapsed before the rat stepped down to the grid floor, was recorded as a measure of the retention of passive avoidance. If the rat did not step down to the grid floor within $2 \mathrm{~min}$, a ceiling score of $2 \mathrm{~min}$ was assigned.

\section{Neurofunctional Assessment}

Motor coordination was evaluated with rotarod tests (Rotamex, Columbus Instruments, Columbus, OH) at PD 26-28 d in DexTD, $\mathrm{HCTD}_{\mathrm{FD}}$, and vehicle groups. Rotarod testing was conducted as previously described with modifications (30). The animals were trained with accelerated speed from 4 to $10 \mathrm{rpm}$ for a maximum of $5 \mathrm{~min}$ at PD26. The rats then were subjected to two trials at PD27 and PD28. The animals were placed in individual lanes with dark Plexiglas spacers between them and the rotarod was set at $25 \mathrm{rpm}$. Latency to first fall was recorded for each animal. For each animal, the trial was completed after $180 \mathrm{~s}$.

\section{Statistical Analysis}

Ordinal variables were compared using Kruskal-Wallis ANOVA on Ranks. Other variables were expressed as mean \pm SEM. The difference was determined by one-way ANOVA and Holm-Sidak method. Sigma Plot 2004 for Windows Version 9.01 (SigmaPlot, Systat Software, San Jose, CA) was used for statistical tests. Differences were considered significant at $P<0.05$.

\section{ACKNOWLEDGMENTS}

We thank Nicole Burton for the admirable English editorial assistance.

\section{STATEMENT OF FINANCIAL SUPPORT}

No financial assistance was received to support this study.

Disclosure: The authors declare no conflicts of interest.

\section{REFERENCES}

1. Barrington KJ. The adverse neuro-developmental effects of postnatal steroids in the preterm infant: a systematic review of RCTs. BMC Pediatr 2001;1:1.

2. Yeh TF, Lin YJ, Lin HC, et al. Outcomes at school age after postnatal dexamethasone therapy for lung disease of prematurity. N Engl J Med 2004;350:1304-13.

3. Flagel SB, Vázquez DM, Watson SJ Jr, Neal CR Jr. Effects of tapering neonatal dexamethasone on rat growth, neurodevelopment, and stress response. Am J Physiol Regul Integr Comp Physiol 2002;282:R55-63.

4. Neal CR Jr, Weidemann G, Kabbaj M, Vázquez DM. Effect of neonatal dexamethasone exposure on growth and neurological development in the adult rat. Am J Physiol Regul Integr Comp Physiol 2004;287:R375-85.

5. van der Heide-Jalving M, Kamphuis PJ, van der Laan MJ, et al. Short- and long-term effects of neonatal glucocorticoid therapy: is hydrocortisone an alternative to dexamethasone? Acta Paediatr 2003;92:827-35.

6. Benders MJ, Groenendaal F, van Bel F, et al. Brain development of the preterm neonate after neonatal hydrocortisone treatment for chronic lung disease. Pediatr Res 2009;66:555-9.

7. Lodygensky GA, Rademaker K, Zimine S, et al. Structural and functional brain development after hydrocortisone treatment for neonatal chronic lung disease. Pediatrics 2005;116:1-7.

8. Halliday HL, Ehrenkranz RA, Doyle LW. Early ( $<8$ days) postnatal corticosteroids for preventing chronic lung disease in preterm infants. Cochrane Database Syst Rev 2010:CD001146.

9. Huang CC, Lin HR, Liang YC, Hsu KS. Effects of neonatal corticosteroid treatment on hippocampal synaptic function. Pediatr Res 2007;62:267-70. 


\section{Articles | Fenget al.}

10. Aden P, Goverud I, Liestøl K, et al. Low-potency glucocorticoid hydrocortisone has similar neurotoxic effects as high-potency glucocorticoid dexamethasone on neurons in the immature chicken cerebellum. Brain Res 2008;1236:39-48.

11. Bhatt AJ, Feng Y, Wang J, Famuyide M, Hersey K. Dexamethasone induces apoptosis of progenitor cells in the subventricular zone and dentate gyrus of developing rat brain. J Neurosci Res 2013;91:1191-202.

12. Kamphuis PJ, Gardoni F, Kamal A, et al. Long-lasting effects of neonatal dexamethasone treatment on spatial learning and hippocampal synaptic plasticity: involvement of the NMDA receptor complex. FASEB J 2003;17:911-3.

13. DeKosky ST, Nonneman AJ, Scheff SW. Morphologic and behavioral effects of perinatal glucocorticoid administration. Physiol Behav 1982;29: 895-900.

14. Jones RA; Collaborative Dexamethasone Trial Follow-up Group. Randomized, controlled trial of dexamethasone in neonatal chronic lung disease: 13- to 17-year follow-up study: I. Neurologic, psychological, and educational outcomes. Pediatrics 2005;116:370-8.

15. Jefferies AL. Postnatal corticosteroids to treat or prevent chronic lung disease in preterm infants. Paediatr Child Health 2012;17:573-4.

16. Watterberg K. Evidence-based neonatal pharmacotherapy: postnatal corticosteroids. Clin Perinatol 2012;39:47-59.

17. Hindmarsh PC. Management of the child with congenital adrenal hyperplasia. Best Pract Res Clin Endocrinol Metab 2009;23:193-208.

18. Peltoniemi O, Kari MA, Heinonen K, et al. Pretreatment cortisol values may predict responses to hydrocortisone administration for the prevention of bronchopulmonary dysplasia in high-risk infants. J Pediatr 2005;146:632-7.

19. Heine VM, Rowitch DH. Hedgehog signaling has a protective effect in glucocorticoid-induced mouse neonatal brain injury through an 11betaHSD2-dependent mechanism. J Clin Invest 2009;119:267-77.
20. Walsh MC, Yao Q, Horbar JD, Carpenter JH, Lee SK, Ohlsson A. Changes in the use of postnatal steroids for bronchopulmonary dysplasia in 3 large neonatal networks. Pediatrics 2006;118:e1328-35.

21. Yoder BA, Harrison M, Clark RH. Time-related changes in steroid use and bronchopulmonary dysplasia in preterm infants. Pediatrics 2009;124: 673-9.

22. Doyle LW, Ehrenkranz RA, Halliday HL. Dexamethasone treatment after the first week of life for bronchopulmonary dysplasia in preterm infants: a systematic review. Neonatology 2010;98:289-96.

23. Watterberg KL, Gerdes JS, Cole CH, et al. Prophylaxis of early adrenal insufficiency to prevent bronchopulmonary dysplasia: a multicenter trial. Pediatrics 2004;114:1649-57.

24. Roberts RS. Early closure of the Watterberg trial. Pediatrics 2004;114: $1670-1$.

25. Gordon PV. Weighing statistical certainty against ethical, clinical, and biologic expediency: the contributions of the Watterberg trial tip the scales in the right direction. Pediatrics 2005;115:1446-7; author reply 1447.

26. Kanagawa T, Tomimatsu T, Hayashi S, et al. The effects of repeated corticosteroid administration on the neurogenesis in the neonatal rat. Am J Obstet Gynecol 2006;194:231-8.

27. Bradford MM. A rapid and sensitive method for the quantitation of microgram quantities of protein utilizing the principle of protein-dye binding. Anal Biochem 1976;72:248-54.

28. Paxinos G, Watson C. The Rat Brain in Stereotaxic Coordinates. 6th edn. Burlington, MA: Academic Press, 2007.

29. Fan LW, Tien LT, Zheng B, Pang Y, Rhodes PG, Cai Z. Interleukin-1betainduced brain injury and neurobehavioral dysfunctions in juvenile rats can be attenuated by alpha-phenyl-n-tert-butyl-nitrone. Neuroscience 2010;168:240-52.

30. Feng Y, Paul IA, LeBlanc MH. Nicotinamide reduces hypoxic ischemic brain injury in the newborn rat. Brain Res Bull 2006;69:117-22. 